\title{
46,XY complete gonadal dysgenesis
}

INSERM

\section{Source}

INSERM. (1999). Orphanet: an online rare disease and orphan drug data base. $\underline{46}$ XY complete gonadal dysgenesis. ORPHA:242

$46, X Y$ complete gonadal dysgenesis $(46, X Y$ CGD) is a disorder of sex development (DSD) associated with anomalies in gonadal development that result in the presence of female external and internal genitalia despite the 46,XY karyotype. 